\title{
Gaps in discrete random samples: extended abstract
}

\author{
Rudolf Grübel ${ }^{1,3}$ \\ Institut für Mathematische Stochastik \\ Leibniz Universität Hannover, Postfach 6009 \\ D-30060 Hannover, Germany \\ Paweł Hitczenko ${ }^{2,4}$ \\ Departments of Mathematics and Computer Science \\ Drexel University \\ 3141 Chestnut Str., Philadelphia PA 19104, USA
}

\begin{abstract}
Motivated by applications in enumerative combinatorics and the analysis of algorithms we investigate the number of gaps and the length of the longest gap in a discrete random sample from a general distribution. We obtain necessary and sufficient conditions on the underlying distribution for the gaps to vanish asymptotically (with probability 1, or in probability), and we study the limiting distributional behavior of these random variables in the geometric case.
\end{abstract}

Keywords: gaps, random samples, geometric distribution.

$\overline{1}$ The first author would like to thank Stephan Klawunn for his pertinent remarks on the first version of the paper.

2 supported in part by NSA Grant \#H98230-09-1-0062

3 Email: rgrubel@stochastik.uni-hannover.de

4 Email: phitczenko@math.drexel.edu 


\section{Introduction}

Let $\left(X_{i}\right)_{i \in \mathbb{N}}$ be a sequence of independent and identically distributed random variables with values in $\mathbb{N}_{0}$, the set of non-negative integers. We assume that

$$
p_{k}:=P\left(X_{1}=k\right)>0 \text { for all } k \in \mathbb{N}_{0} .
$$

We consider $A_{n}:=\left\{X_{1}, \ldots, X_{n}\right\} \subset\left\{m_{n}, \ldots, M_{n}\right\}$ with $M_{n}:=\max _{1 \leq i \leq n} X_{i}$ and $m_{n}:=\min _{1 \leq i \leq n} X_{i}$. By a gap we mean a contiguous and non-empty subset $\{j, \ldots, j+l-1\}$ of the complement $\left\{m_{n}, \ldots, M_{n}\right\} \backslash A_{n}$ of $A_{n}$ in the sample range that is maximal in the sense that both $j-1, j+l \in A_{n}$. We then call $l$ the length of the gap. We are interested in the total number $Y_{n}$ of gaps and the length $L_{n}$ of the longest gap among the first $n$ sample values.

Such quantities are of interest in enumerative combinatorics and the analysis of algorithms; see e.g. [GH07] and the references given there. We also mention connection to infinite urn models and we refer to the recent survey [GHP07] for more information and results. A related concept, weak gaps, essentially the size of $\left\{0, \ldots, M_{n}\right\} \backslash A_{n}$, has been investigated in [LP08]. In all three references the geometric distribution plays a central role.

In this abstract we consider the asymptotic behavior of the number of gaps or the length of the longest gap as the sample size increases to infinity. The results show that the geometric case can be seen as a 'watershed' between $L_{n} \rightarrow 0$ and $L_{n} \rightarrow \infty$. A second aim is the study of the distributional asymptotics of $L_{n}$ as $n \rightarrow \infty$ for geometric random samples. As we will see, the asymptotic distributional periodicities can be resolved in terms of a suitable background construction.

\section{Results}

\subsection{General samples}

Our first two results deal with the extreme case that the gaps will eventually vanish. Let $(\Omega, \mathcal{A}, P)$ be the basic probability space on which the variables $\left(X_{i}\right)_{i \in \mathbb{N}}$ are defined. In view of $L_{n} \in \mathbb{N}_{0}$ the almost sure convergence of $L_{n}$ to 0 as $n \rightarrow \infty$ is equivalent to the property that $L_{n}(\omega)=0$ from some $n=n(\omega)$ onwards, for $P$-almost all $\omega$. Of course, at this end of the spectrum the number of gaps and the length of the longest gap become asymptotically indistinguishable in view of $\left\{Y_{n}=0\right\}=\left\{L_{n}=0\right\}$, so that it is enough to consider one of these variables.

Theorem 2.1 The sequence $\left(L_{n}\right)_{n \in \mathbb{N}}$ converges to 0 with probability 1 as $n \rightarrow$ 
$\infty$ if and only if

$$
\sum_{k=0}^{\infty} \frac{p_{k+1}}{p_{k}}<\infty .
$$

For the weaker convergence in probability we have

Theorem 2.2 Let $Z_{n}$ be $L_{n}$ or $Y_{n}$. Either of the conditions (3) or (4) below is necessary and sufficient for the convergence in probability of $Z_{n}$ to 0 as $n \rightarrow \infty$ :

$$
\begin{aligned}
& \lim _{k \rightarrow \infty} \frac{p_{k+1}}{p_{k}}=0 . \\
& \lim _{n \rightarrow \infty} E Z_{n}=0,
\end{aligned}
$$

Example 2.3 The Poisson distribution with mean $\lambda$ is an example that satisfies (3), but not (2) as

$$
\frac{p_{k+1}}{p_{k}}=\frac{\lambda}{k+1} .
$$

On the other hand, we have the following sufficient condition for the longest gap to converge to $\infty$ in probability.

\section{Theorem 2.4 If}

$$
\lim _{k \rightarrow \infty} \frac{p_{k}}{\sum_{j \geq k} p_{j}}=0
$$

then, for all $l \in \mathbb{N}$,

$$
\lim _{n \rightarrow \infty} P\left(L_{n} \geq l\right)=1
$$

\subsection{Geometric samples}

We now consider the special case that the $X$-sequence is from a geometric distribution: for some $p, 0<p<1$, and all $i \in \mathbb{N}$,

$$
P\left(X_{i}=k\right)=(1-p)^{k} p \quad \text { for all } k \in \mathbb{N}_{0} .
$$

This case plays a central role in the application in enumerative combinatorics and the analysis of algorithms that we mentioned in the introduction.

We write $\mathcal{L}(Y)$ for the distribution of a random quantity $Y$. Our main result below implies that the family $\left\{\mathcal{L}\left(L_{n}\right): n \in \mathbb{N}\right\}$ is tight and that $L_{n_{m}}$ converges in distribution along subsequences $\left(n_{m}\right)_{m \in \mathbb{N}}$ of a specific type determined by $p$. This is a familiar phenomenon in the analysis of random discrete structures and often appears in connection with problems in enumerative combinatorics or the analysis of algorithms. 
Our aim now is to give a probabilistic construction that leads to a representation of the whole family of potential limit distributions along subsequences as deterministic transformations of one single distribution; see [BGr03,Gr07] for more on this approach. Such a construction can be used to handle simultaneously a variety of random variables related to gaps. Below we only deal with $L_{n}$, but the method can also be used for $Y_{n}$. Indeed, the geometric case can be seen as a watershed between the distributions that have an asymptotically contiguous sample range and those where the gaps (number, maximal length) grow beyond all bounds; see Theorem 2.4 above for the latter case. For example, large geometric samples will have one long contiguous part starting at 0 , and our method can be used to analyze the distributional asymptotics of the size

$$
S_{n}:=\max \left\{k \in \mathbb{N}_{0}:\{0,1, \ldots, k\} \subset\left\{X_{1}, \ldots, X_{n}\right\}\right\}
$$

of this block as $n \rightarrow \infty$, or of the difference $M_{n}-S_{n}$.

The starting point for the construction is a sequence $\left(V_{i}\right)_{i \in \mathbb{N}}$ of independent random variables where, for each $i \in \mathbb{N}, V_{i}$ has an exponential distribution with mean $1 / i$. Then

$$
M_{n}^{\prime}:=\max \left\{V_{i}: i=1, \ldots, n-1\right\} \uparrow M_{\infty}:=\sup \left\{V_{i}: i \in \mathbb{N}\right\}
$$

with

$$
P\left(M_{\infty} \leq x\right)=\prod_{k=1}^{\infty}\left(1-e^{-k x}\right) \quad \text { for all } x \geq 0 .
$$

In particular, the maximum of the $V$-variables is finite with probability 1 . Let

$$
W_{l, n}:=\sum_{i=l}^{n} V_{i} \quad \text { for } l \leq n .
$$

It is easy to check that, for all $l \in \mathbb{N}$,

$$
Z_{l, n}:=W_{l, n}-\log n \rightarrow Z_{l, \infty} \quad \text { as } n \rightarrow \infty
$$

almost surely and in quadratic mean for some finite random variable $Z_{l, \infty}$; see e.g. the martingale argument given in [Gr07]. Finally, we define the functions $\phi_{p}:[0, \infty) \rightarrow \mathbb{N}$ and $\psi_{p}:[0, \infty) \rightarrow[0,1)$ by

$$
\phi_{p}(x):=\left\lfloor c(p)^{-1} x\right\rfloor \text { and } \psi_{p}(x):=\left\{c(p)^{-1} x\right\} \text {, }
$$

with

$$
c(p):=-\log (1-p) \text {. }
$$

Here $\{x\}$ denotes the fractional part of $x$; it should be clear from the context whether the curly brackets refer to this function or whether they are used to denote a set. 


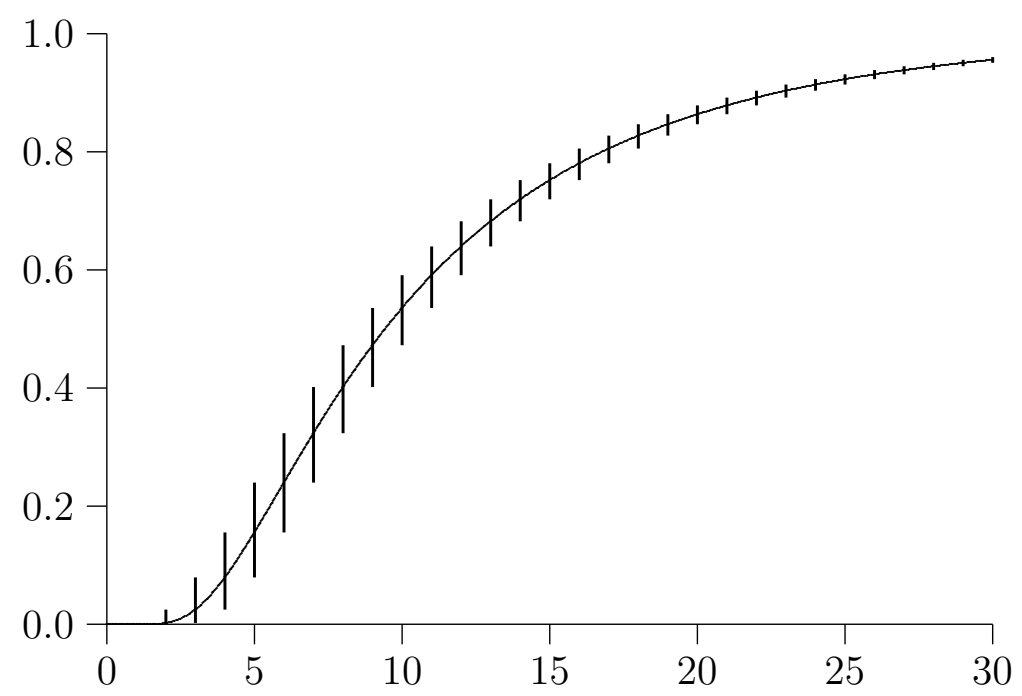

Fig. 1. Continuous limit and discrete bounds (see text)

We can now state our next result. Note that the lower bound in (10) below does not depend on $n$, which implies that $\left\{\mathcal{L}\left(L_{n}\right): n \in \mathbb{N}\right\}$ is tight.

Theorem 2.5 With the notation introduced above,

$$
P\left(M_{\infty} \leq c(p)(l-1)\right) \leq P\left(L_{n} \leq l\right) \leq P\left(M_{n}^{\prime} \leq c(p)(l+1)\right)
$$

for all $n, l \in \mathbb{N}$. Further, if $\left(n_{m}\right)_{m \in \mathbb{N}}$ is such that $n_{m} \rightarrow \infty$ and $\psi_{p}\left(\log n_{m}\right) \rightarrow \eta$ for some $\eta \in[0,1]$ as $m \rightarrow \infty$, then $L_{n_{m}}$ converges in distribution to $L_{\infty}(\eta)$ as $m \rightarrow \infty$, with

$$
L_{\infty}(\eta):=\max _{l \in \mathbb{N}}\left(c(p)^{-1} V_{l}+\psi_{p}\left(Z_{l+1, \infty}+c(p) \eta\right)-\psi_{p}\left(Z_{l, \infty}+c(p) \eta\right)\right) .
$$

Finally, for all $\eta \in[0,1]$ and $l \in \mathbb{N}$,

$$
P\left(M_{\infty} \leq c(p)(l-1)\right) \leq P\left(L_{\infty}(\eta) \leq l\right) \leq P\left(M_{\infty} \leq c(p)(l+1)\right) .
$$

Theorem 2.5 can be used to obtain information about the family of limit distributions. For example, it follows from (11) that, for all $\eta \in[0,1]$,

$$
\left|L_{\infty}(\eta)-c(p)^{-1} M_{\infty}\right| \leq 1 \text {. }
$$

Further (note that we have suppressed the dependence on $p$ in (12)) we see that $p L_{\infty}(\eta)$ converges in distribution to $M_{\infty}$ as $p \rightarrow 0$, whatever $\eta$, which means that for small success probabilities the periodicity will become negligible and which also gives the order of growth of the longest gap. The last statement of the theorem provides upper and lower bounds for the distribution function that arise from shifting the continuous distribution function of $M_{\infty}$, which is given explicitly (and in a numerically accessible form) in (7), 'a bit' 
to the left resp. right. Figure 1 shows the distribution function of $c(p)^{-1} M_{\infty}$ and bounds for $P\left(L_{\infty}(\eta) \leq l\right), 0 \leq \eta \leq 1$, for $p=1 / 10$. As $L_{\infty}(\eta)$ is an integer-valued random variable bounds for its distribution function can be specified by intervals for the values in $l \in \mathbb{N}_{0}$; in the figure, these intervals are visualized by vertical line segments.

Constructions of the above type can be used to obtain an intuitive understanding of the structure of gaps. Clearly, as $n$ increases, a new gap may appear at the right end of the sample, due to a jump in the largest value; nothing may happen at all, due to the fact that the next sample value is already occupied; or an existing gap may shrink or be divided into two smaller gaps. For $p$ small, the limit model may serve as an approximation if, for example, interest is in the probability that the largest gap is the one at the right end. Our last result shows that this happens with probability roughly equal to $1 / 2$.

Theorem 2.6 Let $V_{i}, i \in \mathbb{N}$, be as above and let $M_{l, \infty}:=\max _{i \geq l} V_{i}$. Then, as $p \rightarrow 0$, the limiting probability that the longest gap occurs as the difference of the two largest sample values converges to

$$
P\left(V_{1}>M_{2, \infty}\right)=\int_{0}^{\infty} e^{-x} \prod_{k=2}^{\infty}\left(1-e^{-k x}\right) d x=E M_{1, \infty}-E M_{2, \infty} \approx 0.516 .
$$

\section{References}

[BGr03] Bruss, F. Th. and Grübel, R. (2003) On the multiplicity of the maximum in a discrete random sample. Ann. Appl. Probab. 13, 1252-1263.

[GHP07] Gnedin, A., Hansen, B., and Pitman, J. (2007) Notes on occupancy problems with infinitely many boxes: general asymptotics and power laws, Prob. Surveys 4, 146-171.

[GH07] Goh, W. M. Y. and Hitczenko, P. (2007) Gaps in samples of geometrically distributed random variables, Discrete Math. 307, 2871-2890.

[Gr07] Grübel, R. (2007) Distributional asymptotics in the analysis of algorithms: Periodicities and discretization. Discrete Math. Theor. Comput. Sci. Proc. AH, 451-460.

[HK05] Hitczenko, P. and Knopfmacher, A. (2005) Gap-free compositions and gapfree samples of geometric random variables, Discrete Math. 294, 225-239.

[LP08] Louchard, G. and Prodinger, H. (2008) On gaps and unoccupied urns in sequences of geometrically distributed random variables, Disrete Math. 308, 1538-1562. 